\title{
Resilient Anti-disturbance Dynamic Surface Control of Uncertain Strict-feedback Systems Subject to Partial Loss of Actuator Effectiveness
}

Lu Liu

Dalian Maritime University

Anxin Yang

Guangxi University

Weixing Chen

Shanghai Jiaotong University: Shanghai Jiao Tong University

Weidong Zhang ( 110046@hainanu.edu.cn)

Hainan University

\section{Research Article}

Keywords: Uncertain strict-feedback systems, Partial loss of actuator effectiveness, Dynamic surface control, Data-driven extended state observer

Posted Date: October 26th, 2021

DOl: https://doi.org/10.21203/rs.3.rs-880916/v1

License: (c) (i) This work is licensed under a Creative Commons Attribution 4.0 International License. Read Full License 


\title{
Resilient Anti-disturbance Dynamic Surface Control of Uncertain Strict-feedback Systems Subject to Partial Loss of Actuator Effectiveness
}

\author{
Lu Liu · Anxin Yang - Weixing Chen · Weidong Zhang
}

Received: date / Accepted: date

\begin{abstract}
This paper is concerned with the tracking control of a class of uncertain strict-feedback systems subject to partial loss of actuator effectiveness, in addition to uncertain model dynamics and unknown disturbances. A resilient anti-disturbance dynamic surface control method is proposed to achieve stable tracking regardless of partial actuator faults. First, data-driven adaptive extended state observers are designed based on memory-based identifiers, such that the uncertain model dynamics, external disturbances and the unknown input gains due to actuator faults can be estimated. Next, a resilient anti-disturbance dynamic surface controller is developed based on recovered information from the data-driven adaptive extended state observers. After that, it is proven that the cascade system formed by the observer and controller is input-to-state stable. Finally, comparative studies are performed to validate the efficacy of the resilient anti-disturbance dynamic surface control method for nonlinear strict-feedback systems subject to partial loss of actuator effectiveness.
\end{abstract}

\section{Lu Liu}

School of Marine Engineering, Dalian Maritime University, Dalian 116026, PR China and Department of Automation, Shanghai Jiao Tong University, Shanghai 200240, China.

Anxin Yang

School of Electrical Engineering, Guangxi University, Nanning 530004, PR China.

Weixing Chen

State Key Laboratory of Mechanical System and Vibration, School of Mechanical Engineering, Shanghai Jiao Tong University, Shanghai, China.

Weidong Zhang(Corresponding Author)

School of Information and Communication Engineering, Hainan University, Haikou 570228, Hainan, China and Department of Automation, Shanghai Jiao Tong University, Shanghai 200240, China. E-mail: 110046@hainanu.edu.cn
Keywords Uncertain strict-feedback systems · Partial loss of actuator effectiveness · Dynamic surface control . Data-driven extended state observer

\section{Introduction}

In recent years, the tracking control problem of strictfeedback nonlinear systems has attracted great attention due to its potential applications in many practical engineering systems, such as marine vehicles [1-4], mobile robots [5-7], and aircrafts [8,9]. Since parametric variations, unmodeled dynamics and external disturbances widely exist in a realistic control system, it is highly necessary to develop advanced control methods for uncertain strict-feedback nonlinear systems. During the past few years, various control methods are proposed for strict-feedback nonlinear systems including parametric adaptive control [10], adaptive neural control [11-18], adaptive fuzzy control [19-23], slidingmode control [24], and observer-based control [25-27].

In [10], an adaptive dynamic surface control (DSC) method is developed for uncertain strict-feedback systems, where the nonlinearities are linear in the uncertain model parameters. In [11], a neural network-based adaptive DSC method is developed for uncertain nonlinear strict-feedback systems, and the uncertain parts of the nonlinear systems can be completely unknown. In [19], an adaptive fuzzy DSC approach is developed, where the unknown periodically disturbed functions are compensated by a fuzzy-logic system with a Fourier series expansion. In [20], an output-feedback adaptive fuzzy control method is presented for a class of stochastic nonlinear strict-feedback systems, and this result is extended to uncertain systems with unknown dead zones in [21]. In [12], an input-to-state stability modu- 
lar adaptive neural control method is proposed, and a radial basis function neural network is developed based on partial persistent excitation condition. In [14], an adaptive DSC method is developed by using auxiliary first-order filters, such that the dimension of neural network inputs is reduced. In [13], a composite neural DSC method is presented, and the neural weights are updated using both the prediction error and the compensated tracking error. In [22], a composite adaptive fuzzy controller is proposed for nonlinear strict-feedback systems with unmeasured states and input saturation. In [16], by combining the DSC technology and a disturbance observer, a robust adaptive control scheme is developed for strict-feedback nonlinear systems subject to input saturation and external disturbances. In [15], direct and indirect neural controllers are proposed by using a switching mechanism to feed the transient states back into the neural approximation domain. In [17], a predictor-based neural DSC method is proposed to enable a smooth and fast identification of unknown dynamics without incurring high-frequency oscillations. In [18], an adaptive event-triggered control method is presented, and the neural network weights are aperiodically updated only when the event-triggering condition is violated. In [25], an online recorded data-based neural learning method and nonlinear disturbance observers are cooperating into the neural DSC design. In [24], a robust adaptive learning control method is proposed for uncertain single-input-single-output systems in strictfeedback form by using a switching mechanism. In [26], an adaptive tracking controller is developed for highorder nonlinear strict-feedback systems using a neural network-based state observer. It is worth mentioning that the adaptive controllers in [10-28] do not consider the problem of actuator faults.

Actuators play an important role in linking the control input to the physical actions performed on the system. Normally, the actuators should execute the commands given by the controller faithfully and completely. In this case, the actuators exhibit $100 \%$ effectiveness. However, if a fault occurs in the actuator, the actuator might not be able to complete the control command fully. The actuator effectiveness can be used to measure the severity of the actuator faults. As actuator faults may cause the control performance deterioration or even catastrophic accidents, it is necessary to design a high reliable system in the presence of possible actuator faults. Various adaptive fault-tolerant control methods are proposed for nonlinear systems in [29-35]. In [29], an adaptive fuzzy fault-tolerant DSC method is proposed, and observers are designed to detect, isolate, and estimate the fault. In [30], the dynamic changes induced by actuator faults is considered as the system disturbance and compensated by radial basis function networks. In [31], an adaptive auxiliary signal is designed to compensate for the effect of the actuator fault in nonlinear strict-feedback systems. In [32], an adaptive fuzzy fault-tolerant optimal control method is developed based on an adaptive critic technique. In [33], an event-triggered adaptive fault-tolerant controller are designed, and the effects of actuator faults are compensated by adaptive mechanisms. In [34], the obstacle caused by actuation faults can be compensated by utilizing the approximate property of neural network, and the assumption on control gain matrix is weakened. In [35], a prescribed performance fault-tolerant controller is developed to confine the tracking errors within prescribed arbitrarily small residual sets regardless of actuator faults. Through the above adaptive fault-tolerant controller in [29-35] can compensate for the efforts of actuator faults, the variation of control gains due to actuator faults are still unknown.

Motivated by the above observation, this paper focuses on the tracking control for uncertain strict-feedback systems subject to partial loss of actuator effectiveness, uncertain model dynamics and unknown disturbances. A resilient anti-disturbance DSC method is proposed based on data-driven adaptive extended state observers (DAESOs). Specifically, DAESOs are developed by using a memory-based identifier with a data-driven approach. The DAESOs are able to estimated the unknown input gains caused by actuator faults, in addition to the uncertain model dynamics and external disturbances. Then, based on the proposed DAESOs, a resilient tracking controller is designed without any priori knowledge of model parameters. The observer design is decoupled from the controller design, and the stability of the observer-controller cascade system is analyzed. Simulations results illustrate the effectiveness of the proposed resilient anti-disturbance DSC method based on DAESOs for uncertain strict-feedback systems subject to partial loss of actuator effectiveness.

Compared with the existing adaptive DSC techniques presented in [10-28], and the adaptive fault-tolerant control techniques given in [29-35], main features of the proposed resilient anti-disturbance DSC design are summarized as follows.

- In the adaptive DSC designs in [10-28], the actuator faults are not considered and priori information of control gains is needed. By contrast, the proposed DAESO-based DSC design is able to achieve resilient tracking performance regardless of partial loss of control effectiveness due to the actuator faults.

- In the adaptive fault-tolerant control techniques in [29-35], the efforts of actuator faults can be compensated by adaptive laws, while the variation of control 
gains due to actuator faults are still unknown. By contrast, the unknown control gains due to actuator faults can be accurately estimated by using the proposed DAESOs.

- Compared with the approximation-based adaptive DSC design in [11-23], the proposed DAESO-based resilient anti-disturbance DSC method is easier to implement due to the simplified estimation architecture and decreased tuning parameters.

The rest paper is organized as follows. Section 2 states the problem formulation. Section 3 presents the resilient anti-disturbance dynamic surface controller design including the observer design and the controller design. Section 4 gives the stability analysis of the proposed observer-controller cascade system. Section 5 provides two examples for illustrations. Section 6 concludes this paper.

\section{Problem formulation}

Consider a class of uncertain nonlinear systems in strictfeedback form described as follows

$$
\left\{\begin{array}{l}
\dot{x}_{i}=f_{i}\left(\bar{x}_{i}\right)+w_{i}+b_{i} x_{i+1} \\
\dot{x}_{n}=f_{n}\left(\bar{x}_{n}\right)+w_{n}+b_{n} u \\
y=x_{1}, \quad i=1, \ldots, n-1
\end{array}\right.
$$

where $x_{i} \in \Re$ is the system state; $\bar{x}_{i}=\left[x_{1}, \ldots, x_{i}\right]^{T} \in \Re^{i}$ is the state vector; $u \in \Re$ is the control input; $y \in \Re$ is the system output; $f_{i}\left(\bar{x}_{i}\right)$ is an unknown smooth functions of $\bar{x}_{i}, w_{i} \in \Re$ denotes the external disturbance; $b_{i} \in \Re$ is an unknown control input gain and without loss of generality, it is assumed that $b_{i}>0$. In this paper, the actuator fault is modeled by $b_{n}=\rho b_{o}$ with $b_{o}$ being the unknown control input gain and $0<\rho \leq 1$ being the actuator health indicator. The case $\rho=1 \mathrm{im}-$ plies that the actuator is healthy, and $0<\rho<1$ implies the actuator is partially loses its effectiveness [36].

The control objective is to design an resilient controller for uncertain strict-feedback systems (1) to track a time-varying reference signal $y_{r} \in \Re$ such that the tracking error $z_{1}=y-y_{r}$ converges to a small neighborhood of origin.

In practice, there are many systems falling into the strict-feedback structure, such as the marine ships [3744], underwater vehicles [45-49], mobile robots [50,51], and aircraft $[52,53]$. Therefore the proposed anti-disturbance adaptive DSC design based on DAESOs for uncertain strict-feedback systems can be applied to a wide range of engineering systems in industrial applications.

The following assumptions are needed in the controller design.
Assumption 1. The disturbance $\omega$ and its time derivative $\dot{\omega}$ are bounded, i.e., there exists a positive constant $\epsilon_{1}$ such that $\left|\omega_{i}\right|+\left|\dot{\omega}_{i}\right| \leq \epsilon_{1}$.

Assumption 2. The unknown function and its time derivative are bounded, i.e., there exists a positive constant $\epsilon_{2}$ such that $\left|f_{i}\right|+\left|\frac{\partial f_{i}}{\partial x_{1}}\right|+\left|\frac{\partial f_{i}}{\partial x_{2}}\right|+\ldots+\left|\frac{\partial f_{i}}{\partial x_{i}}\right| \leq \epsilon_{2}$.

Assumption 3. The reference signals $y_{r}, \dot{y}_{r}$ and $\ddot{y}_{r}$ are bounded, i.e., there exists a positive constant $\epsilon_{3}$ such that $y_{r}^{2}+\dot{y}_{r}^{2}+\ddot{y}_{r}^{2} \leq \epsilon_{3}$.

\section{Controller Design}

In this section, a resilient anti-disturbance dynamic surface controller is proposed for uncertain strict-feedback systems subject to partial loss of actuator effectiveness. At first, DAESOs are proposed to estimate the unknown input gains due to actuator faults and the total uncertainties composed of model uncertainties and external disturbances. Then, based on the proposed DAESOs, a resilient anti-disturbance controller is developed using the DSC design method.

\subsection{DAESO Design}

In this subsection, DAESOs are proposed by incorporating memory-based identifiers into the ESOs. The memory-based identifiers are builded based on a concurrent learning technique.

Define the total model uncertainties as

$$
s_{i}=f_{i}\left(\bar{x}_{i}\right)+w_{i},
$$

and it follows that the system model (1) becomes

$$
\left\{\begin{array}{l}
\dot{x}_{i}=s_{i}+b_{i} x_{i+1}, i=1, \ldots, n-1, \\
\dot{x}_{n}=s_{n}+b_{n} u, \\
y=x_{1} .
\end{array}\right.
$$

Let $\hat{x}_{i}$ be the estimates of $x_{i}$ with $i=1, \ldots, n . \hat{s}_{i}$ represents the estimates of $s_{i}$ with $i=1, \ldots, n$. $\hat{b}_{i}$ donates the estimates of $b_{i}$ with $i=1, \ldots, n$. The estimation errors are defined as follows

$\left\{\begin{array}{l}\tilde{x}_{i}=\hat{x}_{i}-x_{i}, \\ \tilde{s}_{i}=\hat{s}_{i}-s_{i}, \\ \tilde{b}_{i}=\hat{b}_{i}-b_{i} .\end{array}\right.$

For the $i$ th subsystem $\dot{x}_{i}=s_{i}+b_{i} x_{i+1}$, a DAESO is proposed as follows

$\left\{\begin{array}{l}\dot{\hat{x}}_{i}=-k_{i 1}\left(\hat{x}_{i}-x_{i}\right)+\hat{s}_{i}+\hat{b}_{i} x_{i+1}, \\ \dot{\hat{s}}_{i}=-k_{i 2}\left(\hat{x}_{i}-x_{i}\right),\end{array}\right.$

where $i=1, \ldots n$ and $x_{n+1}=u ; k_{i 1}$ and $k_{i 2}$ are positive observer gains, which can be selected as $k_{i 1}=\omega_{c}^{2}, k_{i 2}=$ $2 \omega_{c}$ with $\omega_{c}$ being the control band-width. 
The update law for the input gains $\hat{b}_{i}$ is designed by using the past stored and current data concurrently as follows

$$
\left\{\begin{aligned}
\dot{\dot{x}}_{i} & =\hat{s}_{i}+\hat{b}_{i} x_{i+1}-k_{i m} \bar{x}_{i}+m_{i} \dot{\hat{b}}_{i}, \\
\dot{\hat{b}}_{i} & =k_{i c} \operatorname{Proj}\left\{\hat{b}_{i},-m_{i}\left(\bar{x}_{i}-\gamma_{i}\right)\right. \\
& \left.-\sum_{k=1}^{p} m_{i k}\left(\bar{x}_{i k}-\gamma_{i k}\right)\right\}, \\
\dot{m}_{i} & =x_{i+1}-k_{i m} m_{i},
\end{aligned}\right.
$$

where $k_{i m} \in \Re$ and $k_{i c} \in \Re$ are positive scalar gains; $m_{i k} \in \Re, \bar{x}_{i k} \in \Re$ and $\gamma_{i k} \in \Re$ are the stored data of $m_{i}$, $\bar{x}_{i}$ and $\gamma_{i}$ at different time instants $t=t_{k} ; \bar{x}_{i}=\check{x}_{i}-x_{i}$. Here $\gamma_{i}$ is defined as

$\gamma_{i}=\bar{x}_{i}-m_{i} \tilde{b}_{i}, \gamma_{i}\left(t_{0}\right)=\bar{x}_{i}\left(t_{0}\right)$,

and $\operatorname{Proj}[\cdot]$ is a projection operator which is used to guarantee that $0<b_{\text {imin }} \leq \hat{b}_{i} \leq b_{\text {imax }}$.

Note that

$\left\{\begin{array}{l}\dot{\bar{x}}_{i}=\tilde{s}_{i}+\tilde{b}_{i} x_{i+1}-k_{i m} \bar{x}_{i}+m_{i} \dot{\tilde{b}}_{i}, \\ \dot{m}_{i} \tilde{b}_{i}=\tilde{b}_{i} x_{i+1}-k_{i m} m_{i} \tilde{b}_{i}\end{array}\right.$

and the derivative of $\gamma_{i}$ can be described as

$\dot{\gamma}_{i}=-k_{i m} \gamma_{i}+\tilde{s}_{i}$.

Since $\gamma_{i}$ is unavailable, let $\hat{\gamma}_{i}$ be an estimation of $\gamma_{i}$ updated as follows

$\dot{\hat{\gamma}}_{i}=-k_{i m} \hat{\gamma}_{i}, \hat{\gamma}_{i}\left(t_{0}\right)=\bar{x}_{i}\left(t_{0}\right)$

Let

$\tilde{\gamma}_{i}=\hat{\gamma}_{i}-\gamma_{i}$

then it can be derived that

$\dot{\tilde{\gamma}}_{i}=-k_{i m} \tilde{\gamma}_{i}-\tilde{s}_{i}$

By using $\hat{\gamma}_{i}$, the update law in (5) becomes

$$
\begin{array}{r}
\dot{\hat{b}}_{i}=k_{i c} \operatorname{Proj}\left\{\hat{b}_{i},-m_{i}\left(\bar{x}_{i}-\hat{\gamma}_{i}\right)\right. \\
\left.-\sum_{k=1}^{p} m_{i k}\left(\bar{x}_{i k}-\hat{\gamma}_{i k}\right)\right\},
\end{array}
$$

where $\hat{\gamma}_{i k}$ is the stored data of $\hat{\gamma}_{i}$ at different time instants $t=t_{k}$.
The dynamics of $\tilde{b}_{i}$ is expressed by

$$
\begin{aligned}
\dot{\tilde{b}}_{i} & =k_{i c} \operatorname{Proj}\left\{\hat{b}_{i},-m_{i}\left(\bar{x}_{i}-\gamma_{i}-\tilde{\gamma}_{i}\right)\right. \\
& \left.-\sum_{k=1}^{p} m_{i k}\left(\bar{x}_{i k}-\gamma_{i k}-\tilde{\gamma}_{i k}\right)\right\} \\
& =k_{i c} \operatorname{Proj}\left\{\hat{b}_{i},-m_{i}\left(m_{i} \tilde{b}_{i}-\tilde{\gamma}_{i}\right)\right. \\
& \left.-\sum_{k=1}^{p} m_{i k}\left(m_{i k} \tilde{b}_{i}-\tilde{\gamma}_{i k}\right)\right\} \\
& =k_{i c} \operatorname{Proj}\left\{\hat{b}_{i},-m_{i}^{2} \tilde{b}_{i}-\sum_{k=1}^{p} m_{i k}^{2} \tilde{b}_{i}\right. \\
& \left.+m_{i} \tilde{\gamma}_{i}+\sum_{k=1}^{p} m_{i k} \tilde{\gamma}_{i k}\right\},
\end{aligned}
$$

where $\tilde{\gamma}_{i k}=\hat{\gamma}_{i k}-\gamma_{i k}$.

To move on, the following assumption is required.

Assumption 4. The stored data of the proposed DAESOs satisfies that $\sum_{k=1}^{p} m_{i k}^{2}>0$.

Take the time derivative of $\tilde{x}_{i}$ and $\tilde{s}_{i}$ using (4) and (2), and the error dynamics of the DAESOs can be expressed as

$\left\{\begin{array}{l}\dot{\tilde{x}}_{i}=-k_{i 1} \tilde{x}_{i}+\tilde{s}_{i}+\tilde{b}_{i} x_{i+1}, \\ \dot{\tilde{s}}_{i}=-k_{i 2} \tilde{x}_{i}-\dot{s}_{i}, \\ \dot{\tilde{\gamma}}_{i}=-k_{i m} \tilde{\gamma}_{i}-\tilde{s}_{i},\end{array}\right.$

and it can be written in a compact form

$\dot{X}_{i}=A_{i} X_{i}+\sigma_{i}$,

with $X_{i}=\left[\tilde{x}_{i}, \tilde{s}_{i}, \tilde{\gamma}_{i}\right]^{T}$ and

$A_{i}=\left[\begin{array}{ccc}-k_{i 1} & 1 & 0 \\ -k_{i 2} & 0 & 0 \\ 0 & -1 & -k_{i m}\end{array}\right], \sigma_{i}=\left[\begin{array}{c}\tilde{b}_{i} x_{i+1} \\ -\dot{s}_{i} \\ 0\end{array}\right]$.

In the parameter estimation methods presented in [54], the unknown model parameters including the input gains can be estimated accurately, but they cannot deal with the modeling errors and external disturbances. By using the proposed DAESOs, the internal model uncertainties, external disturbances and the unknown control gains due to actuator faults can be estimated simultaneously.

Different from the classic ESOs proposed in [5568] where the input gains are assumed to be known, a DAESO is developed herein. With the aid of a memorybased identifier, the DAESO is able to estimate the unknown control gains in addition to the total disturbances. 


\subsection{DSC control Design}

In this subsection, a resilient anti-disturbance controller is designed based on the proposed DAESOs and a DSC method. As a result, the system is able to track the reference signal against the partial loss of actuator effectiveness without any knowledge of model parameters. In the following text, the controller is designed step by step.

The uncertain strict-feedback systems in (2) can be rewritten as

$$
\left\{\begin{array}{l}
\dot{x}_{i}=s_{i}-\tilde{b}_{i} x_{i+1}+\hat{b}_{i} x_{i+1}, i=1, \ldots, n-1, \\
\dot{x}_{n}=s_{n}-\tilde{b}_{n} u+\hat{b}_{n} u, \\
y=x_{1} .
\end{array}\right.
$$

Step 1: Let

$z_{1}=x_{1}-y_{r}$

and take the time derivative of $z_{1}$

$\dot{z}_{1}=\hat{b}_{1} x_{2}-\tilde{b}_{1} x_{2}+s_{1}-\dot{y}_{r}$.

The virtual control law is given by

$\alpha_{2}=\frac{1}{\hat{b}_{1}}\left(-k_{1} z_{1}-\hat{s}_{1}+\dot{y}_{r}\right)$,

with $k_{1}$ being a positive constant. Let $\alpha_{2}$ pass through a first-order filter to obtain its estimate, denoted by $\alpha_{2 d}$

$\xi_{1} \dot{\alpha}_{2 d}+\alpha_{2 d}=\alpha_{2}, \alpha_{2 d}(0)=\alpha_{2}(0)$,

where $\xi_{1}$ is a constant.

Step $i$ : Define

$z_{i}=x_{i}-\alpha_{i d}$

then the time derivative of $z_{i}$ can be written as

$\dot{z}_{i}=\hat{b}_{i} x_{i+1}-\tilde{b}_{i} x_{i+1}+s_{i}-\dot{\alpha}_{i d}$.

Construct a virtual control law as

$\alpha_{i+1}=\frac{1}{\hat{b}_{i}}\left(-k_{i} z_{i}-\hat{s}_{i}+\dot{\alpha}_{i d}\right)$,

with $k_{i}$ being a positive constant. Let $\alpha_{i+1}$ pass through a first-order filter to obtain $\alpha_{(i+1) d}$

$\xi_{i} \dot{\alpha}_{(i+1) d}+\alpha_{(i+1) d}=\alpha_{i+1}, \alpha_{(i+1) d}(0)=\alpha_{i+1}(0)$,

where $\xi_{i}$ is a constant.

Step $n$ : Let

$z_{n}=x_{n}-\alpha_{n d}$,

and take the time derivative of $z_{n}$

$\dot{z}_{n}=\hat{b}_{n} u-\tilde{b}_{n} u+s_{n}-\dot{\alpha}_{n d}$.
The control law $u$ is designed as follows

$u=\frac{1}{\hat{b}_{n}}\left(-k_{n} z_{n}-\hat{s}_{n}+\dot{\alpha}_{n d}\right)$,

where $k_{n}$ is a positive constant.

From the control design above, the closed-loop system can be described by

$\left\{\begin{array}{l}\dot{z}_{1}=-k_{1} z_{1}+\hat{b}_{1} z_{2}+\hat{b}_{1} q_{2}-\tilde{s}_{1}-\tilde{b}_{1} x_{2}, \\ \quad \vdots \\ \dot{z}_{i}=-k_{i} z_{i}+\hat{b}_{i} z_{i+1}+\hat{b}_{i} q_{i+1}-\tilde{s}_{i}-\tilde{b}_{i} x_{i+1}, \\ \quad \vdots \\ \dot{z}_{n}=-k_{n} z_{n}-\tilde{s}_{n}-\tilde{b}_{n} u\end{array}\right.$

where $q_{i+1}=\alpha_{(i+1) d}-\alpha_{i+1}, i=1, \ldots, n-1$.

Take the time derivatives of $q_{i+1}$ yields

$$
\left\{\begin{aligned}
\dot{q}_{2} & =-\frac{q_{2}}{\xi_{1}}-\frac{\dot{\hat{b}}_{1}}{\hat{b}_{1}^{2}}\left(-k_{1} z_{1}-\hat{s}_{1}+\dot{y}_{r}\right) \\
& -\frac{1}{\hat{b}_{1}}\left(-k_{1} \dot{z}_{1}-\dot{\hat{s}}_{1}+\ddot{y}_{r}\right), \\
& =-\frac{q_{2}}{\xi_{1}}+B_{2}\left(z_{1}, z_{2}, q_{2}, \tilde{s}_{1}, \tilde{x}_{1}, \hat{b}_{1}, \tilde{b}_{1}, y_{r}, \dot{y}_{r}, \ddot{y}_{r}\right) \\
\vdots & \\
\dot{q}_{i+1} & =-\frac{q_{i+1}}{\xi_{i}}-\frac{\dot{\hat{b}}_{i}}{\hat{b}_{i}^{2}}\left(-k_{i} z_{i}-\hat{s}_{i}+\dot{\alpha}_{i d}\right) \\
& -\frac{1}{\hat{b}_{i}}\left(-k_{i} \dot{z}_{i}-\hat{\hat{s}}_{i}+\ddot{\alpha}_{i d}\right), \\
& =-\frac{q_{i+1}}{\xi_{i}}+B_{i+1}\left(z_{1}, \ldots z_{i+1}, q_{2}, \ldots q_{i+1}, \tilde{s}_{1}, \ldots, \tilde{s}_{i},\right. \\
& \left.\tilde{x}_{1}, \ldots, \tilde{x}_{i}, \hat{b}_{1}, \ldots, \hat{b}_{i}, \tilde{b}_{1}, \ldots, \tilde{b}_{i}, y_{r}, \dot{y}_{r}, \ddot{y}_{r}\right) \\
\vdots & \\
& =-\frac{q_{n}}{\xi_{n-1}}-\frac{\dot{\hat{b}}_{n-1}}{\hat{b}_{n-1}^{2}}\left(-k_{n-1} z_{n-1}-\hat{s}_{n-1}+\dot{\alpha}_{i d}\right) \\
& -\frac{1}{\dot{b}_{n-1}}\left(-k_{n-1} \dot{z}_{n-1}-\dot{\hat{s}}_{n-1}+\ddot{\alpha}_{(n-1) d}\right) \\
& =-\frac{q_{n}}{\xi_{n-1}}+B_{n}\left(z_{1}, \ldots z_{n}, q_{2}, \ldots q_{n},\right. \\
& \tilde{s}_{1}, \ldots, \tilde{s}_{n-1}, \tilde{x}_{1}, \ldots, \tilde{x}_{n-1}, \hat{b}_{1}, \ldots, \hat{b}_{n-1}, \\
& \left.\tilde{b}_{1}, \ldots, \tilde{b}_{n-1}, y_{r}, \dot{y}_{r}, \ddot{y}_{r}\right),
\end{aligned}\right.
$$

with $B_{2}(\cdot), \ldots, B_{n}(\cdot)$ being continuous functions.

A visualization of the proposed resilient anti-disturbance control architecture is shown in Fig. 1. It consists of two parts, namely, data-driven adaptive ESOs and a resilient anti-disturbance dynamic surface controller. In the data-driven adaptive ESOs, a memory-based identifier based on recorded data is combined with ESOs to identify the model uncertainty and unknown input gains. By using the estimated information from the DAESOs, a resilient anti-disturbance dynamic surface controller is designed step by step without any priori knowledge of the model parameters. 


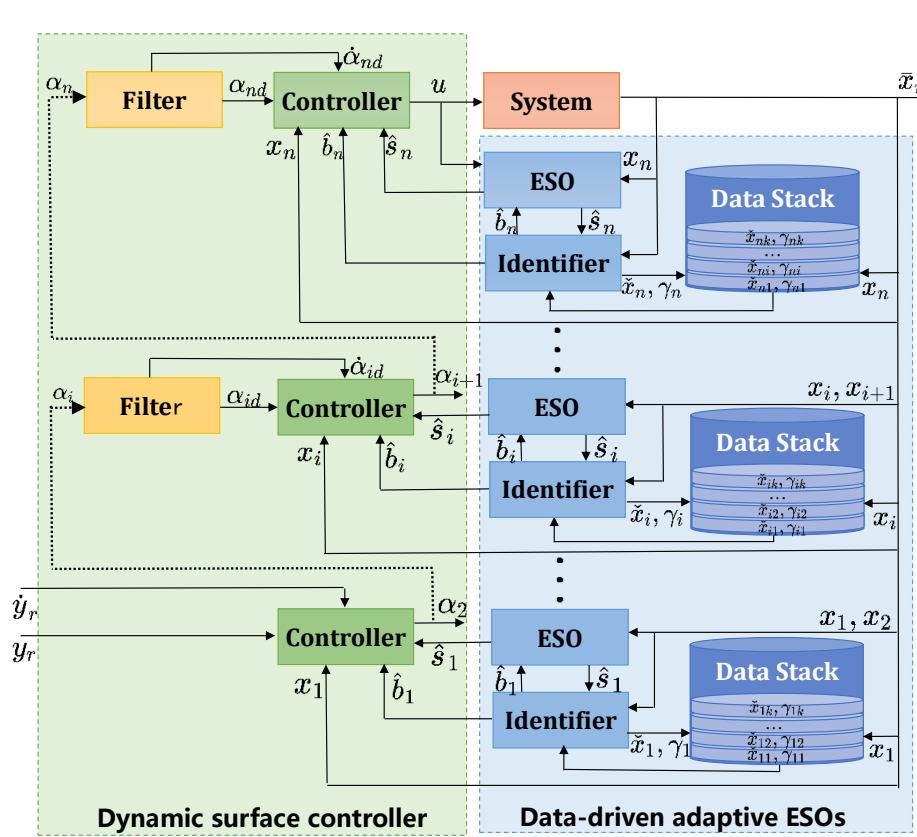

Fig. 1 Resilient anti-disturbance DSC architecture based on DAESO.

\section{Stability Analysis}

\subsection{Stability analysis of the DAESOs}

In this section, the stability of the proposed DAESO for resilient anti-disturbance dynamic surface controller is analyzed. First, the stability of the ESO subsystem (15) is analyzed.

According to (15), the full error dynamic can be rewritten as

$\dot{X}=A X+\sigma$,

where $X=\left[X_{1}, \ldots, X_{n}\right]^{T}, A=\operatorname{diag}\left\{A_{1}, \ldots, A_{n}\right\}, \sigma=$ $\left[\sigma_{1}, \ldots, \sigma_{n}\right]^{T}$.

Here $A$ are Hurwitz, i.e., there exists a positive definite symmetric matrix $P$ satisfies the Lyapunov equation

$P A+A^{T} P=-I$.

Lemma 1. If there exits a positive definite matrix $P$ satisfying (32) and Assumptions 1 and 2 are satisfied, then the system (31) with the state vector being $X$ and the inputs $\tilde{b}_{1}, \ldots, \tilde{b}_{n}$ and $\dot{s}_{1}, \ldots, \dot{s}_{n}$ is input-to-state stable (ISS).

Proof : Consider a Lyapunov candidate as

$V_{1}=\frac{1}{2} X^{T} P X$.

The time derivative of $V_{1}$ along (31) is expressed by

$\dot{V}_{1}=\frac{1}{2} X^{T}\left(P A+A^{T} P\right) X+X^{T} P \sigma$.
Using (32), it follows that

$\dot{V}_{1}=-\frac{1}{2}\|X\|^{2}+\|X\|\|P\|\|\sigma\|$.

Let $x_{n+1}=u$, and note that

$$
\begin{aligned}
\|X\| & \geq \frac{2\|P\|\left(\sum_{i=1}^{n}\left\|x_{i+1}\right\|\left\|\tilde{b}_{i}\right\|+\sum_{i=1}^{n}\left\|s_{i}\right\|\right)}{\bar{\theta}_{1}} \\
& \geq \frac{2\|P\| \sqrt{\sum_{i=1}^{n}\left\|x_{i+1}\right\|^{2}\left\|\tilde{b}_{i}\right\|^{2}+\sum_{i=1}^{n}\left\|s_{i}\right\|^{2}}}{\bar{\theta}_{1}} \\
& \geq \frac{2\|P\|\|\sigma\|}{\bar{\theta}_{1}},
\end{aligned}
$$

with $0<\bar{\theta}_{1}<1$ renders

$\dot{V}_{1} \leq-\frac{1-\bar{\theta}_{1}}{2}\|X\|^{2}$.

The boundness of $\tilde{b}_{i}$ is guaranteed by projection operation, and the upper bound for $\tilde{b}_{i}$ is given by $\left\|\tilde{b}_{i}\right\| \leq$ $2 b^{*}+\varepsilon$. According to Assumptions 1 and 2, there exists a positive constant $s^{*}$ such that $\left\|\dot{s}_{i}\right\| \leq s^{*}$. Then we can conclude that the subsystem (31) with respect to the input $\tilde{b}_{1}, \ldots, \tilde{b}_{n}$ and $\dot{s}_{1}, \ldots, \dot{s}_{n}$, is ISS, and $\|X\|$ is bounded by

$$
\begin{array}{r}
\|X(t)\| \leq \max \left\{\varpi_{1}\left(\left\|X\left(t_{0}\right)\right\|\right), \sum_{i=1}^{n} \phi^{\tilde{b}_{i}}\left(\tilde{b}_{i}\right)\right. \\
\left.+\sum_{i=1}^{n} \phi^{\dot{s}_{i}}\left(\dot{s}_{i}\right)\right\}, \forall t \geq t_{0},
\end{array}
$$

where $\varpi_{1}$ is a $\mathcal{K} \mathcal{L}$ function and

$\left\{\begin{array}{l}\phi^{\tilde{b}_{i}}(\iota)=\sqrt{\frac{\lambda_{\max }(P)}{\lambda_{\min }(P)}} \frac{2\|P\|\left\|x_{i+1}\right\|\|\iota\|}{\bar{\theta}_{1}}, \\ \phi^{\dot{s}_{i}}(\iota)=\sqrt{\frac{\lambda_{\max }(P)}{\lambda_{\min }(P)}} \frac{2\|P\| \iota \iota \|}{\theta_{1}} .\end{array}\right.$

The proof of Lemma 1 is completed.

Next, the stability of identification subsystem (13) is analyzed.

Lemma 2. The subsystem (13) with the state vector being $\tilde{b}_{1}, \ldots, \tilde{b}_{n}$ and the input vector being $\tilde{\gamma}_{1}, \ldots, \tilde{\gamma}_{n}$ is ISS.

Proof. Construct the Lyapunov function candidate as

$V_{2}=\sum_{i=1}^{n} \frac{1}{2 k_{i c}} \tilde{b}_{i}^{2}$.

Take the time derivative of $V_{2}$, one has

$$
\begin{aligned}
\dot{V}_{2}= & \sum_{i=1}^{n}\left\{-m_{i}^{2} \tilde{b}_{i}^{2}-\sum_{k=1}^{p} m_{i k}^{2} \tilde{b}_{i}^{2}\right. \\
& \left.+m_{i} \tilde{b}_{i} \tilde{\gamma}_{i}+\sum_{k=1}^{p} m_{i k} \tilde{b}_{i} \tilde{\gamma}_{i k}\right\} \\
= & \sum_{i=1}^{n}\left\{-k_{i b} \tilde{b}_{i}^{2}+m_{i} \tilde{b}_{i} \tilde{\gamma}_{i}+\sum_{k=1}^{p} m_{i k} \tilde{b}_{i} \tilde{\gamma}_{i k}\right\},
\end{aligned}
$$


where $k_{i b}=m_{i}^{2}+\sum_{k=1}^{p} m_{i k}^{2}>0$.

Since

$\left|\tilde{b}_{i}\right| \geq \frac{\left|m_{i}\right|\left|\tilde{\gamma}_{i}\right|+\sum_{k=1}^{p}\left|m_{i k}\right|\left|\tilde{\gamma}_{i k}\right|}{\bar{\theta}_{i 2} k_{i b}}$,

with $0<\bar{\theta}_{i 2}<1$ renders

$\dot{V}_{2} \leq \sum_{i=1}^{n}\left\{-k_{i b}\left(1-\bar{\theta}_{i 2}\right)\left|\tilde{b}_{i}\right|^{2}\right\}$

Then we can conclude that the subsystem (13) with respect to the input $\tilde{\gamma}_{i}$, is ISS, and $\tilde{b}_{i}$ is bounded by

$\left|\tilde{b}_{i}\right| \leq \max \left\{\varpi_{i 2}\left(\tilde{b}_{i}\left(t_{0}\right)\right), \phi^{\tilde{\gamma}_{i}}\left(\tilde{\gamma}_{i}\right)+\sum_{k=1}^{p} \phi^{\tilde{\gamma}_{i k}}\left(\tilde{\gamma}_{i k}\right)\right\}$

$\forall t \geq t_{0}$, where $\varpi_{i 2}$ is a $\mathcal{K} \mathcal{L}$ function and

$\left\{\begin{array}{l}\phi^{\tilde{\gamma}_{i}}(\iota)=\frac{\left|m_{i}\right||\iota|}{\bar{\theta}_{i 2} k_{i b}}, \\ \phi^{\tilde{\gamma}_{i k}}(\iota)=\frac{\left|m_{i k}\right||\iota|}{\theta_{i 2} k_{i b}} .\end{array}\right.$

The proof of Lemma 2 is completed.

Note that the subsystems (31) and (13) form a cascade system, where the identification subsystem (13) is excited by the ESO subsystem (31). The stability of the cascade system is indicated as follows.

Lemma 3. Consider the strict-feedback uncertain system (1), the DAESO (4), the data-driven identifier (5). Under Assumptions 1 and 2, the cascade system form by the subsystems (31) and (13) is ISS.

Proof: Lemma 1 shows that the subsystem (31) with the state vector being $X$ and the input vector being $\tilde{b}_{1}, \ldots, \tilde{b}_{n}$ and $\dot{s}_{1}, \ldots, \dot{s}_{n}$ is ISS. Lemma 2 shows that the identification subsystem (13) with the state being $\tilde{b}_{1}, \ldots, \tilde{b}_{n}$ and the inputs being $\tilde{\gamma}_{1}, \ldots, \tilde{\gamma}_{n}$ is ISS. According to Lemma C.4 in [69], it can be concluded that the cascade system (31) and (13) is ISS with the states being $X, \tilde{b}_{1}, \ldots, \tilde{b}_{n}$, and input being $\tilde{b}_{1}, \ldots, \tilde{b}_{n}$ and $\dot{s}_{1}, \ldots, \dot{s}_{n}$ is ISS. Define $Y_{1}=\left[X^{T}, \tilde{b}_{1}, \ldots, \tilde{b}_{n}\right]^{T}$, then $Y_{1}$ is bounded by

$$
\begin{aligned}
\left\|Y_{1}(t)\right\| \leq \max \left\{\varpi_{3}\left(\left\|Y_{1}(0)\right\|, t\right),\right. \\
\left.\sum_{i=1}^{n} \phi_{i 1}\left(\tilde{b}_{i}\right)+\sum_{i=1}^{n} \phi_{i 2}\left(\dot{s}_{i}\right)\right\}, \forall t \geq t_{0},
\end{aligned}
$$

where $\varpi_{3}$ is a class $\mathcal{K} \mathcal{L}$ function and $\phi_{i 1}$ and $\phi_{i 2}$ are class $\mathcal{K}$ functions.
4.2 Stability analysis of the dynamic surface controller

In this subsection, the stability of the resilient antidisturbance dynamic surface controller is analyzed. The following lemma presents the stability of tracking error subsystem (29) and (30).

Lemma 4. The error subsystem (29) and (30) with the states being $z_{1}, \ldots, z_{n}, q_{2}, \ldots, q_{n}$, and the inputs being $\tilde{s}_{1}, \ldots, \tilde{s}_{n}, \tilde{b}_{1}, \ldots, \tilde{b}_{n}$ and $B_{2}, \ldots, B_{n}$ is ISS.

Proof : Construct a Lyapunov function as follows

$V_{3}=\sum_{i=1}^{n} \frac{1}{2} z_{i}^{2}+\sum_{i=1}^{n-1} \frac{1}{2} q_{i+1}^{2}$

whose derivative along (29) and (30) satisfies

$$
\begin{aligned}
\dot{V}_{3} & =-\sum_{i=1}^{n} k_{i} z_{i}^{2}-\sum_{i=1}^{n-1} \hat{b}_{i} z_{i+1} z_{i}-\sum_{i=1}^{n-1} \hat{b}_{i} q_{i+1} z_{i} \\
& -\sum_{i=1}^{n-1} \frac{1}{\xi_{i}} q_{i+1}^{2}+Z^{T} h,
\end{aligned}
$$

with

$\left\{\begin{array}{l}Z=\left[z_{1}, \ldots, z_{n}, q_{2}, \ldots, q_{n}\right]^{T}, \\ h=\left[-\tilde{s}_{1}-\tilde{b}_{1} x_{2}, \ldots,-\tilde{s}_{n}-\tilde{b}_{n} u, B_{2}, \ldots, B_{n}\right]^{T} .\end{array}\right.$

Using

$\left\{\begin{array}{l}\hat{b}_{i} z_{i+1} z_{i} \leq \frac{\left(b^{*}+\varepsilon\right) z_{i+1}^{2}}{2}+\frac{\left(b^{*}+\varepsilon\right) z_{i}^{2}}{2}, \\ \hat{b}_{i} q_{i+1} z_{i} \leq \frac{\left(b^{*}+\varepsilon\right) q_{i+1}^{2}}{2}+\frac{\left(b^{*}+\varepsilon\right) z_{i}^{2}}{2} .\end{array}\right.$

it follows that

$\dot{V}_{3} \leq Z^{T} K Z+Z^{T} h$,

with $K=\operatorname{diag}\left\{-\left[k_{1}-\left(b^{*}+\varepsilon\right)\right], \ldots,-\left[k_{i}-3\left(b^{*}+\varepsilon\right) / 2\right], \ldots,-\left[k_{n}-\right.\right.$ $\left.\left(b^{*}+\varepsilon\right) / 2\right],-\left[1 / \xi_{1}-\left(b^{*}+\varepsilon\right) / 2, \ldots,-\left[1 / \xi_{n-1}-\left(b^{*}+\varepsilon\right) / 2\right]\right\}$.

As

$$
\begin{aligned}
\|Z\| & \geq \frac{\sum_{i=1}^{n}\left|\tilde{s}_{i}\right|}{\bar{\theta}_{3} \lambda_{\min }(K)}+\frac{\sum_{i=1}^{n}\left|x_{i+1}\right|\left|\tilde{b}_{i}\right|}{\bar{\theta}_{3} \lambda_{\min }(K)} \\
& +\frac{\sum_{i=1}^{n-1}\left|B_{i+1}\right|}{\bar{\theta}_{3} \lambda_{\min }(K)}
\end{aligned}
$$

with $0<\bar{\theta}_{3}<1$ renders

$\dot{V}_{3} \leq-\left(1-\bar{\theta}_{3}\right) \lambda_{\min }(K)\|Z\|^{2}$.

Therefore, the error subsystem (29) is ISS and $\|Z\|$ is bounded by

$$
\begin{array}{r}
\|Z(t)\| \leq \max \left\{\varpi_{4}\left(Z\left(t_{0}\right)\right), \sum_{i=1}^{n} \phi^{\tilde{s}_{i}}\left(\tilde{s}_{i}\right)\right. \\
\left.+\sum_{i=1}^{n} \phi^{\tilde{b}_{i}}\left(\tilde{b}_{i}\right)+\sum_{i=1}^{n-1} \phi^{B_{i+1}}\left(B_{i+1}\right)\right\},
\end{array}
$$


$\forall t \geq t_{0}$, where $\varpi_{4}$ is a $\mathcal{K} \mathcal{L}$ function and

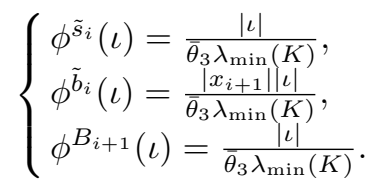

The proof of Lemma 4 is completed.

\subsection{Cascade Stability Analysis}

The cascade system consists of the subsystem (31), (13), (29) and (30), where the tracking error subsystem (29) and (30) is excited by the DAESO subsystem (31) and (13). The stability of the cascade system is indicated as follows.

Theorem 1. Consider the strict-feedback uncertain system (1), the DAESO (4), the data-driven identifier (5), together with the virtual control laws (20) (24) and the control law (28). Under Assumptions 1, 2 and 3, the cascade system form by the subsystems (31), (13), (29) and (30) is ISS.

Proof: Lemma 3 shows that the cascade system (31) and (13) is ISS with the states being $X, \tilde{b}_{1}, \ldots, \tilde{b}_{n}$, and inputs being $\tilde{b}_{1}, \ldots, \tilde{b}_{n}$ and $\dot{s}_{1}, \ldots, \dot{s}_{n}$. Lemma 4 shows that the subsystem (29) and (30) with the states being $z_{1}, \ldots, z_{n}, q_{2}, \ldots, q_{n}$, and the inputs being $\tilde{s}_{1}, \ldots, \tilde{s}_{n}$, $\tilde{b}_{1}, \ldots, \tilde{b}_{n}, B_{2}, \ldots, B_{n}$ is ISS. According to Lemma C.4 in [69], it can be concluded that the cascade system (31) and (29) is ISS with the states being $X, \tilde{b}_{1}, \ldots, \tilde{b}_{n}$, $z_{1}, \ldots, z_{n}, q_{2}, \ldots, q_{n}$, and inputs being $\tilde{b}_{1}, \ldots, \tilde{b}_{n}, \dot{s}_{1}, \ldots, \dot{s}_{n}$ and $B_{2}, \ldots, B_{n}$.

Note that $\left\|\tilde{b}_{i}\right\| \leq 2 b^{*}+\varepsilon$ and $\left\|\dot{s}_{i}\right\| \leq s^{*}$. Since for $\varpi_{0} \leq 0$ and $V_{1}(0)+V_{2}(0)+V_{3}(0) \leq \varpi_{1}$, the sets $\prod_{1}=$ $\left\{y_{r}^{2}+\dot{y}_{r}^{2}+\ddot{y}_{r}^{2} \leq \varpi_{0}\right\}$ and $\Pi_{2}=\left\{X^{T} P X+\sum_{i=1}^{n} \tilde{b}_{i}^{2} / k_{i c}+\right.$ $\left.\sum_{i=1}^{n} z_{i}^{2}+\sum_{i=1}^{n-1} q_{i+1}^{2}\right\} \leq 2 \varpi_{1}$ are compact. Therefore, there exists positive constants $B^{*}$ such that $\left\|B_{i+1}\right\| \leq$ $B^{*}, i=1, \ldots, n-1$.

Define $Y_{2}=\left[X^{T}, \tilde{b}_{1}, \ldots, \tilde{b}_{n}, z_{1}, \ldots, z_{n}, q_{2}, \ldots, q_{n}\right]^{T}$, then $Y_{2}$ is bounded by

$$
\begin{aligned}
\left\|Y_{2}(t)\right\| & \leq \max \left\{\varpi_{5}\left(\left\|Y_{2}(0)\right\|, t\right), \sum_{i=1}^{n} \phi_{i 3}\left(\tilde{b}_{i}\right)\right. \\
& \left.+\sum_{i=1}^{n} \phi_{i 4}\left(\dot{s}_{i}\right)+\sum_{i=1}^{n-1} \phi_{i 5}\left(B_{i+1}\right)\right\}, \forall t \geq t_{0},
\end{aligned}
$$

where $\varpi_{5}$ is a class $\mathcal{K} \mathcal{L}$ function and $\phi_{i 3}, \phi_{i 4}$ and $\phi_{i 5}$ are class $\mathcal{K}$ functions.

\section{Illustrative examples}

Two examples are provided to verify the performance of the proposed resilient anti-disturbance DSC method based on DAESOs.
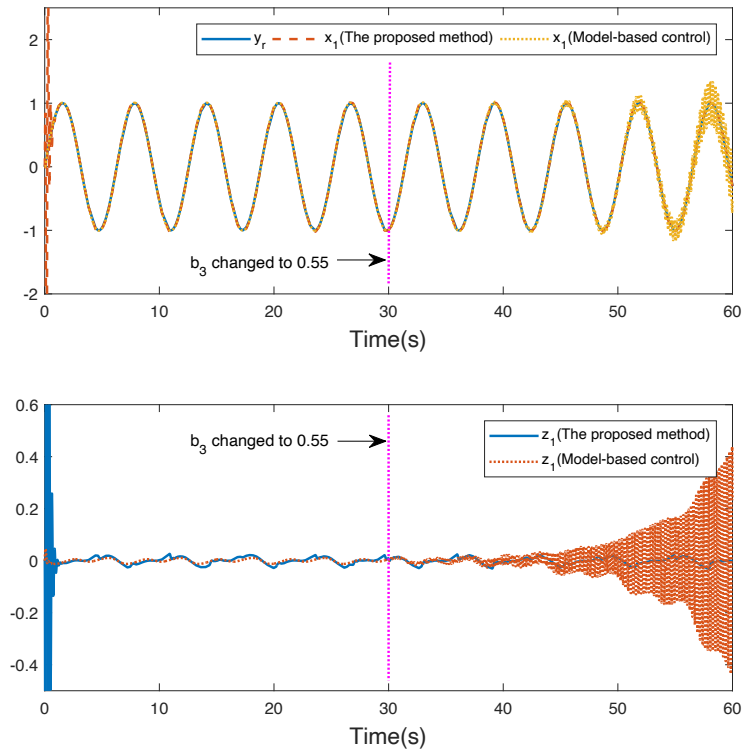

Fig. 2 Comparisons of the the proposed method and the model-based control method.
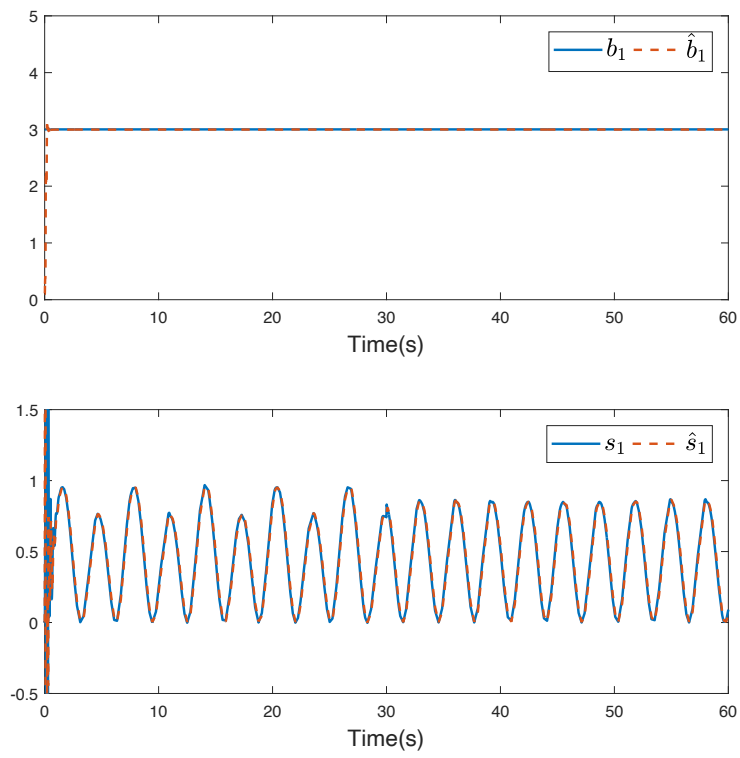

Fig. 3 Estimation performance of the data-driven adaptive ESO.
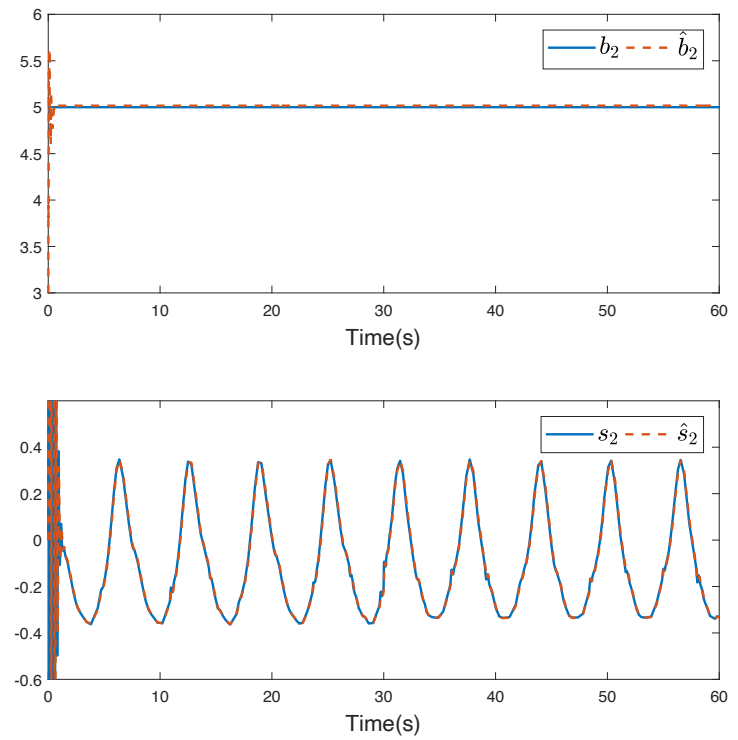

Fig. 4 Estimation performance of the data-driven adaptive ESO. 
Example 1. Consider a third-order uncertain nonlinear system in strict-feedback form as

$$
\left\{\begin{array}{l}
\dot{x}_{1}=x_{1} \sin x_{1}+w_{1}+b_{1} x_{2} \\
\dot{x}_{2}=x_{2} \cos x_{1}+w_{2}+b_{2} x_{3} \\
\dot{x}_{3}=x_{1} x_{3}+x_{2}^{2}+x_{3} \sin x_{2}+w_{3}+b_{3} u
\end{array}\right.
$$

where $w_{1}, w_{2}, w_{3}$ are external disturbances; $b_{1}, b_{2}, b_{3}$ are unknown control input gains. Let $s_{1}=x_{1} \sin x_{1}+w_{1}$, $s_{2}=x_{2} \cos x_{1}+w_{2}$ and $s_{3}=x_{1} x_{3}+x_{2}^{2}+x_{3} \sin x_{2}+w_{3}$, which denote the model uncertainties.

The control parameters are chosen as $k_{1}=5, k_{2}=$ $50, k_{3}=50, \xi_{1}=0.05, \xi_{2}=0.05, k_{o 1}=90, k_{o 2}=2025$, $k_{o 3}=100, k_{o 4}=2500, k_{o 5}=120, k_{o 6}=3600, k_{m 1}=$ $200, k_{c 1}=1500, k_{m 2}=500, k_{c 2}=2000, k_{m 3}=350$, $k_{c 3}=22000$. The reference signal is selected as $y_{r}=$ $\sin (t)$.

To illustrate, the proposed resilient anti-disturbance DSC method based on DAESO is compared with a model-based control method. The model-based controller is given as:

$$
\left\{\begin{array}{l}
\alpha_{2}=\frac{1}{b_{1}}\left(-k_{1} z_{1}-s_{1}+\dot{y}_{r}\right), \\
\xi_{1} \dot{\alpha}_{2 d}+\alpha_{2 d}=\alpha_{2}, \alpha_{2 d}(0)=\alpha_{2}(0), \\
\alpha_{3}=\frac{1}{b_{2}}\left(-k_{2} z_{2}-s_{2}+\dot{\alpha}_{2 d}\right) \\
\xi_{2} \dot{\alpha}_{3 d}+\alpha_{3 d}=\alpha_{3}, \alpha_{3 d}(0)=\alpha_{3}(0), \\
u=\frac{1}{b_{3}}\left(-k_{3} z_{3}-s_{3}+\dot{\alpha}_{3 d}\right) .
\end{array}\right.
$$

Simulation results are illustrated in Figs. 2-5. Fig. 2 depicts the comparisons of the proposed method and the model-based control method. The reference signal and the outputs of the two methods are shown in the first subfigure of Fig. 2. The tracking errors of the two methods are plotted in the second subfigure of Fig. 2. It can be observed that during the time interval $[0,30]$, the tracking performance of the proposed method and the model-based control method is almost the same. However, during the time interval [30,60], the modelbased control method suffers from a poor tracking performance since it cannot capture the variations of control gains. Fig. 3-Fig. 5 shows the estimation profile of the unknown control inputs gains and the total disturbances composed of model uncertainty and external disturbances. They demonstrate that the uncertainties are efficiently compensated by the proposed DAESOs. Especially, in Fig. 5, it can be observed that both the control inputs gains and the total disturbances can be reconstructed regardless of the variation of control input gain.

Example 2. The yaw dynamics of a marine surface vehicle can be described by

$$
\left\{\begin{array}{l}
\dot{\psi}=r \\
\dot{r}=-\frac{\kappa}{\Gamma} \mathrm{H}(r)+\frac{\kappa}{\Gamma} \delta
\end{array}\right.
$$
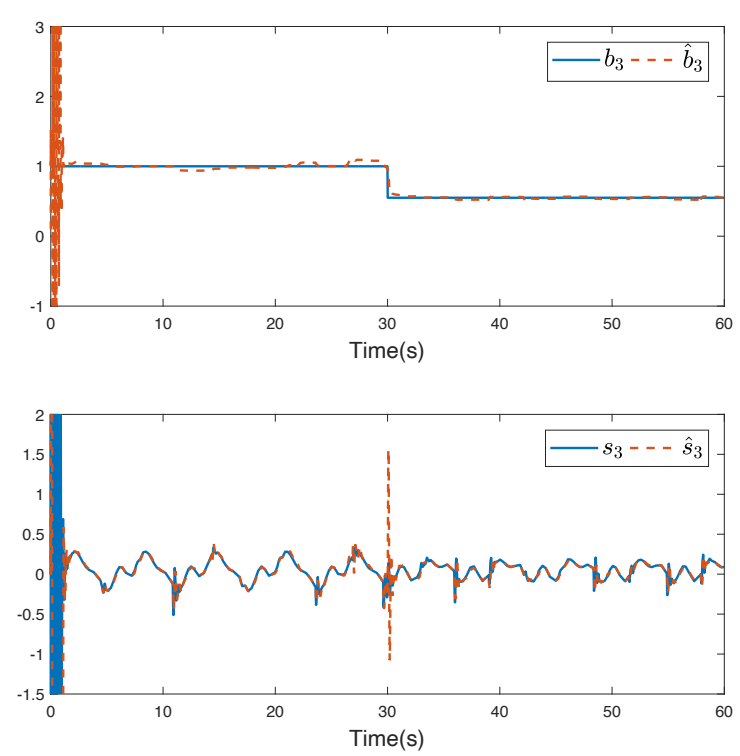

Fig. 5 Estimation performance of the data-driven adaptive ESO.
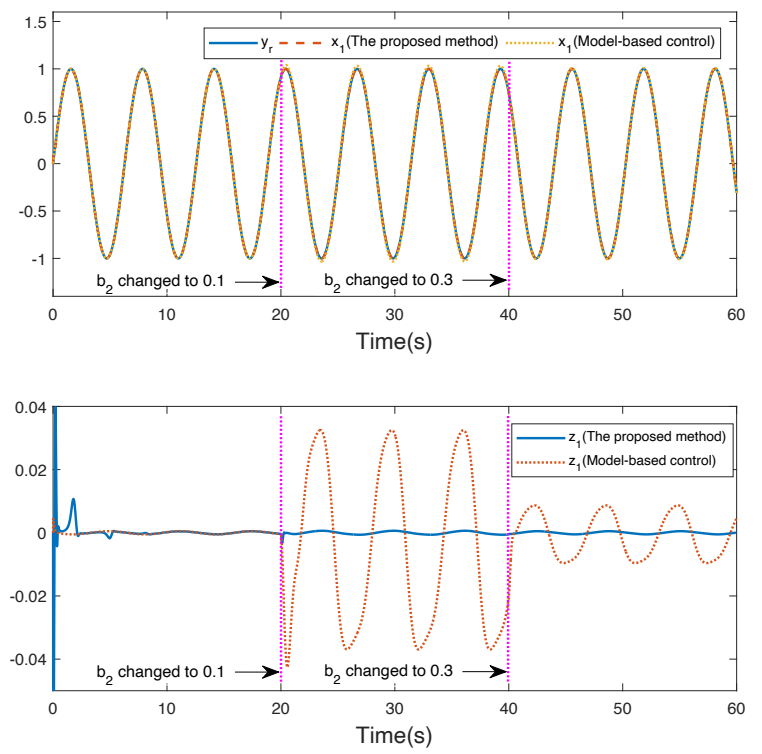

Fig. 6 Comparisons of the the proposed method and the model-based control method.
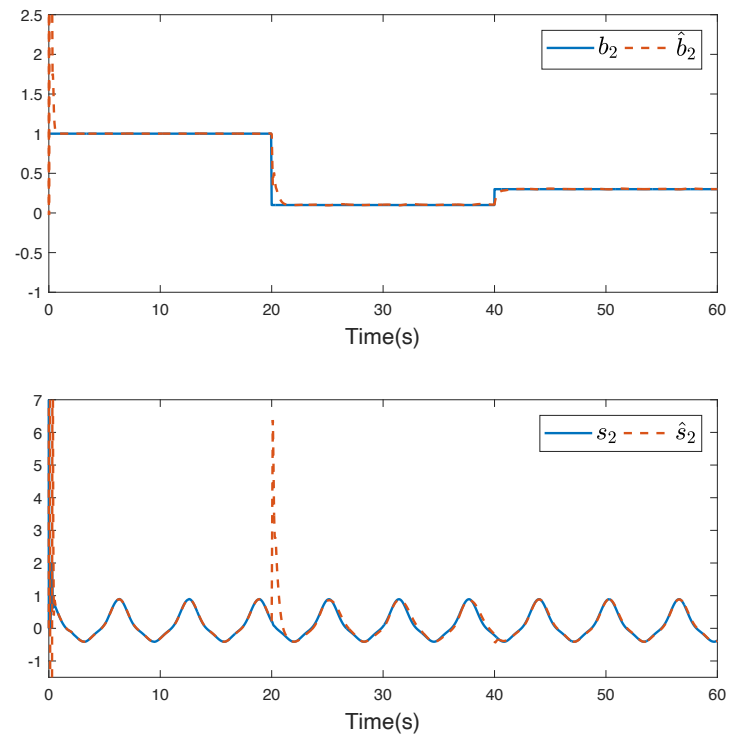

Fig. 7 Estimation performance of the data-driven adaptive ESO. 
where $\psi$ is the yaw angle, $r$ is the yaw rate, $\delta$ is the actuator angle; $\mathrm{H}(r)$ is an unknown nonlinear function of $r ; \kappa$ and $\Gamma$ are unknown hydrodynamic coefficients. Let $x_{1}=\psi, x_{2}=r, s_{2}=-(\kappa / \Gamma) \mathrm{H}(r), b_{2}=\kappa / \Gamma, u=$ $\delta$, and then, the yaw dynamic (59) can be transformed into the form of (2).

The control parameters are chosen as $k_{1}=5, k_{2}=$ $30, \xi_{1}=0.005, k_{o 3}=160, k_{o 4}=6400, k_{m 1}=10, k_{c 1}=$ $5000, k_{m 2}=200, k_{c 2}=8000$. The reference signal is selected as $y_{r}=\sin (t)$.

The proposed controller is compared with the following model-based controller

$$
\left\{\begin{array}{l}
\alpha_{2}=-k_{1} z_{1}+\dot{y}_{r} \\
\xi_{1} \dot{\alpha}_{2 d}+\alpha_{2 d}=\alpha_{2}, \alpha_{2 d}(0)=\alpha_{2}(0) \\
u=\frac{1}{b_{2}}\left(-k_{2} z_{2}-s_{2}+\dot{\alpha}_{2 d}\right)
\end{array}\right.
$$

Simulation results are illustrated in Figs. 6-7. Fig. 6 plots the comparison results of the tracking performance of yaw angle and tracking errors by using the proposed method and the model-based control method. It can be observed that if the control input gain is fixed, small tracking errors can be achieved by both the proposed control method and the model-based control method. However, if the control input gain is changed, the model-based control method suffers from large tracking errors due to the fact that it cannot adapt to the parameter variations. Fig. 7 displays the model uncertainties and the outputs of DAESO, and the control input gain is changed at $20 \mathrm{~s}$ and $40 \mathrm{~s}$. It indicates that the unknown control input gain and uncertain nonlinear term are efficiently compensated by the DAESO.

\section{Conclusion}

In this paper, a resilient anti-disturbance DSC design approach is presented for uncertain strict-feedback systems subject to partial loss of actuator effectiveness, in addition to uncertain model dynamics and unknown disturbances. In the estimator design, DAESOs are proposed to estimate the unknown input gains caused by actuator faults, as well as the uncertain model dynamics and external disturbances. In the controller design, a resilient dynamic surface controller is proposed based on the DAESOs, such that the partial loss of actuator effectiveness can be compensated. In addition, no priori knowledge of model parameters is needed in the controller design. Rigorous analysis shows that the cascade system is ISS. Simulation and comparison results validated the performance improvement of the resilient anti-disturbance DSC method based on DAESOs in the presence of partial loss of actuator effectiveness.

\section{Acknowledgement}

This work was supported in part by the National Natural Science Foundation of China under Grants 51979020 , 51909021, 51939001, 52071044, and in part by China Postdoctoral Science Foundation 2019M650086, and in part by the Fundamental Research Funds for the Central Universities under Grant 3132019319.

\section{Declarations}

Conflict of interest The authors declare that they have no conflict of interest.

Data availability The data sets generated during and analyzed during the current study are available from the corresponding authors on reasonable request.

\section{References}

1. Y.-L. Wang and Q.-L. Han, "Network-based heading control and rudder oscillation reduction for unmanned surface vehicles," IEEE Transactions on Control Systems Technology, vol. 25, no. 5, pp. 1609-1620, 2017.

2. Y.-L. Wang, Q.-L. Han, M.-R. Fei, and C. Peng, "Network-based T-S fuzzy dynamic positioning controller design for unmanned marine vehicles," IEEE Transactions on Cybernetics, vol. 48, no. 9, pp. 27502764, 2018.

3. Z. Peng, J. Wang, D. Wang, and Q. Han, "An overview of recent advances in coordinated control of multiple autonomous surface vehicles," IEEE Transactions on Industrial Informatics, 2020, DOI: 10.1109/TII.2020.3004343.

4. Z. Peng, D. Wang, T. Li, and M. Han, "Output feedback cooperative formation maneuvering of autonomous surface vehicles with connectivity preservation and collision avoidance," IEEE Transactions on Cybernetics, vol. 50, no. 6, pp. 2527-2535, Jun. 2020.

5. B. Ning, Q.-L. Han, and Q. Lu, "Fixed-time leaderfollowing consensus for multiple wheeled mobile robots," IEEE Transactions on Cybernetics, vol. 50, no. 10, pp. 4381-4392, 2020.

6. E. Mousavinejad, X. Ge, Q.-L. Han, T. J. Lim, and L. Vlacic, "An ellipsoidal set-membership approach to distributed joint state and sensor fault estimation of autonomous ground vehicles," IEEE/CAA Journal of Automatica Sinica, vol. 8, no. 6, pp. 1107-1118, 2021.

7. D. Zhang, Q.-L. Han, and X.-M. Zhang, "Network-based modeling and proportional-integral control for directdrive-wheel systems in wireless network environments," IEEE Transactions on Cybernetics, vol. 50, no. 6, pp. 2462-2474, 2020.

8. B. $\mathrm{Xu}$, "Disturbance observer-based dynamic surface control of transport aircraft with continuous heavy cargo airdrop," IEEE Transactions on Systems, Man, and Cybernetics: Systems, vol. 47, no. 1, pp. 161-170, Jan. 2017.

9. M. Chen, P. Shi, and C.-C. Lim, "Adaptive neural faulttolerant control of a 3-DOF model helicopter system," IEEE Transactions on Systems, Man, and Cybernetics: Systems, vol. 46, no. 2, pp. 260-270, Feb 2016. 
10. P. P. Yip and J. K. Hedrick, "Adaptive dynamic surface control: A simplified algorithm for adaptive backstepping control of nonlinear systems," International Journal of Control, vol. 71, no. 5, pp. 959-979, Jan. 1998.

11. D. Wang and J. Huang, "Neural network-based adaptive dynamic surface control for a class of uncertain nonlinear systems in strict-feedback form," IEEE Transactions on Neural Networks, vol. 16, no. 1, pp. 195-202, Jan. 2005.

12. C. Wang, M. Wang, T.-F. Liu, and D. J. Hill, "Learning from ISS-modular adaptive NN control of nonlinear strict-feedback systems," IEEE Transactions on Neural Network Learning System, vol. 23, no. 10, pp. 1539-1550, Oct. 2012.

13. B. Xu, Z. Shi, C. Yang, and F. Sun, "Composite neural dynamic surface control of a class of uncertain nonlinear systems in strict-feedback form," IEEE Transactions on Cybernetics, vol. 44, no. 12, pp. 2626-2634, Dec. 2014.

14. M. Wang and C. Wang, "Learning from adaptive neural dynamic surface control of strict-feedback systems," IEEE Transactions on Neural Networks and Learning Systems, vol. 26, no. 6, pp. 1247-1259, Jun. 2015.

15. B. Xu, C. Yang, and Y. Pan, "Global neural dynamic surface tracking control of strict-feedback systems with application to hypersonic flight vehicle," IEEE Transactions on Neural Networks and Learning Systems, vol. 41, no. 6, pp. 2563-2575, Oct. 2015.

16. M. Chen, G. Tao, and B. Jiang, "Dynamic surface control using neural networks for a class of uncertain nonlinear systems with input saturation," IEEE Transactions on Neural Network Learning System, vol. 26, no. 9, pp. 2086-2097, Sep. 2015.

17. Z. Peng, D. Wang, and J. Wang, "Predictor-based neural dynamic surface control for uncertain nonlinear systems in strict-feedback form," IEEE Transactions on Neural Networks and Learning Systems, vol. 28, no. 9, pp. 21562167, Sep. 2016.

18. Y. Li and G. Yang, "Model-based adaptive eventtriggered control of strict-feedback nonlinear systems," IEEE Transactions on Neural Networks and Learning Systems, vol. 29, no. 4, pp. 1033-1045, Apr. 2018.

19. W. Chen, L. Jiao, R. Li, and J. Li, "Adaptive backstepping fuzzy control for nonlinearly parameterized systems with periodic disturbances," IEEE Transactions on Fuzzy Systems, vol. 18, no. 4, pp. 674-685, Aug. 2010.

20. S. Tong, Y. Li, G. Feng, and T. Li, "Observer-based adaptive fuzzy backstepping dynamic surface control for a class of MIMO nonlinear systems," IEEE Transactions on Systems, Man, and Cybernetics, Part B (Cybernetics), vol. 41, no. 4, pp. 1124-1135, Aug. 2011.

21. S. Tong and Y. Li, "Adaptive fuzzy output feedback tracking backstepping control of strict-feedback nonlinear systems with unknown dead zones," IEEE Transactions on Fuzzy Systems, vol. 20, no. 1, pp. 168-180, Feb 2012.

22. Y. Li, S. Tong, and T. Li, "Composite adaptive fuzzy output feedback control design for uncertain nonlinear strictfeedback systems with input saturation," IEEE Transactions on Cybernetics, vol. 45, no. 10, pp. 2299-2308, Oct. 2015.

23. H. Wang, W. Bai, X. Zhao, and P. X. Liu, "Finite-timeprescribed performance-based adaptive fuzzy control for strict-feedback nonlinear systems with dynamic uncertainty and actuator faults," IEEE Transactions on $C y$ bernetics, Jan. 2021, DOI: 10.1109/TCYB.2020.3046316.

24. B. Xu, X. Wang, W. Chen, and P. Shi, "Robust intelligent control of SISO nonlinear systems using switching mech- anism," IEEE Transactions on Cybernetics, Apr. 2020, DOI: 10.1109/TCYB.2020.2982201.

25. B. Xu, Y. Shou, J. Luo, H. Pu, and Z. Shi, "Neural learning control of strict-feedback systems using disturbance observer," IEEE Transactions on Neural Networks and Learning Systems, vol. 30, no. 5, pp. 1296-1307, Sep. 2019.

26. C. L. P. Chen, G.-X. Wen, Y.-J. Liu, and Z. Liu, "Observer-based adaptive backstepping consensus tracking control for high-order nonlinear semi-strict-feedback multiagent systems," IEEE Transactions on Cybernetics, vol. 46, no. 7, pp. 1591-1601, Jul. 2016

27. J. Qiu, T. Wang, K. Sun, I. J. Rudas, and H. Gao, "Disturbance observer-based adaptive fuzzy control for strict-feedback nonlinear systems with finite-time prescribed performance," IEEE Transactions on Fuzzy Systems, Jan. 2021, DOI: 10.1109/TFUZZ.2021.3053327.

28. R. Cui, J. Guo, and Z. Mao, "Adaptive backstepping control of wheeled inverted pendulums models," Nonlinear Dynamics, vol. 79, no. 1, pp. 501-511, Sep. 2014.

29. Q. Shen, B. Jiang, and V. Cocquempot, "Adaptive fuzzy observer-based active fault-tolerant dynamic surface control for a classof nonlinear systems with actuator faults," IEEE Transcations on Fuzzy Systems, vol. 22, no. 2, pp. 338-349, 2014

30. K. A. Semprun, L. Yan, W. A. Butt, and P. C. Y. Chen, "IEEE transactions on neural networks and learning systems," Dynamic Surface Control for a Class of Nonlinear FeedbackLinearizable Systems With Actuator Failures, vol. 28, no. 9, pp. 2209-2214, 2017.

31. Z. Wang, L. Liu, Y. Wu, and H. Zhang, "Optimal faulttolerant control for discrete-timenonlinear strict-feedback systems basedon adaptive critic design," IEEETransactions on Neural Network and Learning Systems, vol. 29, no. 6, pp. 2179-2191, 2018.

32. Y. Li, S. Member, K. Sun, and S. Tong, "Observerbased adaptive fuzzy fault-tolerantoptimal control for siso nonlinear systems," IEEE Transactions on Cybernetics, vol. 49, no. 2, pp. 649-661, 2019.

33. C.-H. Zhang and G.-H. Yang, "Event-triggered adaptive output feedback controlfor a class of uncertain nonlinear systemswith actuator failures," IEEE Transactions on Cybernetics, vol. 50, no. 1, pp. 201-210, 2020.

34. K. Zhao and J. Chen, "Adaptive neural quantized control of mimononlinear systems under actuation faults andtime-varying output constraints," IEEETransactions on Neural Network and Learning Systems, vol. 31, no. 9, pp. 3471-3481, 2020.

35. Y. Nai, M. Qingyu Yang, and Z. Wu, "Prescribed performance adaptive neuralcompensation control for intermittentactuator faults by state andoutput feedback," IEEE Transactions on Neural Networks and Learning Systems, 2021, dOI:10.1109/TCYB.2020.3046316.

36. B. Xiao, Q. Hu, and P. Shi, "Attitude stabilization of spacecrafts under actuator saturation and partial loss of control effectiveness," IEEE Transactions on Control Systems Technology, vol. 21, no. 6, pp. 2251-226., 2013.

37. S. Dai, S. He, and C. Wang, "Platoon formation control with prescribed performance guarantees for USVs," IEEE Transactions on Industrial Electronics, vol. 65, no. 5, pp. 4237-4246, May 2018.

38. S.-L. Dai, S. He, M. Wang, and C. Yuan, "Adaptive neural control of underactuated surface vessels with prescribed performance guarantees," IEEE Transactions on Neural Networks and Learning Systems, vol. 30, no. 12, pp. 3686-3698, Dec. 2019. 
39. Z. Peng, L. Liu, and J. Wang, "Output-feedback flocking control of multiple autonomous surface vehicles based on data-driven adaptive extended state observers," IEEE Transactions on Cybernetics, Aug. 2020, DOI:10.1109/TCYB.2020.3009992.

40. L. Liu, D. Wang, Z. Peng, T. Li, and C. P. Chen, "Cooperative path following ring-networked under-actuated autonomous surface vehicles: Algorithms and experimental results," IEEE transactions on Cybernetics, vol. 50, no. 4, pp. 1519-1529, Apr. 2020.

41. Z. Peng, D. Wang, and J. Wang, "Data-driven adaptive disturbance observers for model-free trajectory tracking control of maritime autonomous surface ships," IEEE Transactions on Neural Networks and Learning Systems, Jul. 2021, DOI: 10.1109/TNNLS.2021.3093330.

42. Z. Peng, J. Wang, and D. Wang, "Distributed maneuvering of autonomous surface vehicles based on neurodynamic optimization and fuzzy approximation," IEEE Transactions on Control Systems Technology, vol. 26, no. 3, pp. 1083-1090, May 2018.

43. — , "Distributed containment maneuvering of multiple marine vessels via neurodynamics-based output feedback," IEEE Transactions on Industrial Electronics, vol. 65, no. 4, pp. 3831-3839, May 2017.

44. L. Liu, D. Wang, and Z. Peng, "Direct and composite iterative neural control for cooperative dynamic positioning of marine surface vessels," Nonlinear Dynamics, vol. 81, no. 3, pp. 1315-1328, Aug. 2015.

45. Z. Peng, J. Wang, and J. Wang, "Constrained control of autonomous underwater vehicles based on command optimization and disturbance estimation," IEEE Transactions on Industrial Electronics, vol. 66, no. 5, pp. 36273635, Jul. 2019.

46. Z. Peng, J. Wang, and Q.-L. Han, "Path-following control of autonomous underwater vehicles subject to velocity and input constraints via neurodynamic optimization," IEEE Transactions on Industrial Electronics, vol. 66, no. 11 , pp. $8724-8732$, Nov. 2019.

47. R. Cui, W. Yan, and D. Xu, "Synchronization of multiple autonomous underwater vehicles without velocity measurements," Science China Information Sciences, vol. 55, no. 7, pp. 1693-1703, Apr 2012.

48. R. Cui, X. Zhang, and D. Cui, "Adaptive sliding-mode attitude control for autonomous underwater vehicles with input nonlinearities," Ocean Engineering, vol. 123, pp. 45-54, Sep. 2016.

49. Z.-H. Peng, D. Wang, H. Wang, and W. Wang, "Distributed coordinated tracking of multiple autonomous underwater vehicles," Nonlinear Dynamics, vol. 78, pp. 1261-1276, Jul. 2014.

50. M. Chen, "Robust tracking control for self-balancing mobile robots using disturbance observer," IEEE/CAA Journal of Automatica Sinica, vol. 4, no. 3, pp. 458-465, Jul. 2017.

51. N. Gu, Z. Peng, D. Wang, and F. Zhang, "Path-guided containment maneuvering of mobile robots: Theory and experiments," IEEE Transactions on Industrial Electronics, vol. 68, no. 8, pp. 7178-7187, 2020.

52. M. Chen, B. Ren, Q. Wu, and C. Jiang, "Antidisturbance control of hypersonic flight vehicles with input saturation using disturbance observer," Science China Information Sciences, vol. 58, no. 7, pp. 1-12, May 2015.

53. M. Lv, Y. Li, W. Pan, and S. Baldi, "Finite-time fuzzy adaptive constrained tracking control for hypersonic flight vehicles with singularity-free switching,"
IEEE/ASME Transactions on Mechatronics, Jun. 2021 DOI: 10.1109/TMECH.2021.3090509.

54. S. B. Roy, S. Bhasin, and I. N. Kar, "Combined mrac for unknown mimo lti systems with parameter convergence," IEEE Transactions on Automatica Control, vol. 63 , no. 1 , pp. 283-290, Jan. 2018.

55. J. Han, "From pid to active disturbance rejection control," IEEE Transactions on Industrial Electronics, vol. 56, pp. 900-906, Mar. 2009.

56. D. Yoo, S. S. Yau, and Z. Gao, "Optimal fast tracking observer bandwidth of the linear extended state observer," International Journal of Control, vol. 80, no. 1, pp. 102111, Apr. 2007.

57. B. Guo and Z. Zhao, "On the convergence of an extended state observer for nonlinear systems with uncertainty," Systems \& Control Letters, vol. 60, no. 6, pp. 420-430 Jun. 2011.

58. Z. Zhao and B. Guo, "Extended state observer for uncertain lower triangular nonlinear systems," Systems $\mathcal{E}$ Control Letters, vol. 85, pp. 100-108, Nov. 2015.

59. X. L. Shao and H. L. Wang, "Back-stepping active disturbance rejection control design for integrated missile guidance and control system via reduced-order ESO," ISA Transactions, vol. 57, pp. 10-20, Jul. 2015.

60. Z. Zhao and B. Guo, "A nonlinear extended state observer based on fractional power functions," Automatica, vol. 81, p. 286-296, Jul. 2017.

61. M. Fu and L. Yu, "Finite-time extended state observerbased distributed formation control for marine surface vehicles with input saturation and disturbances," Ocean Engineering, vol. 159, pp. 219-227, Jul. 2018.

62. L. Liu, D. Wang, and Z. Peng, "State recovery and disturbance estimation of unmanned surface vehicles based on nonlinear extended state observers," Ocean Engineering, vol. 171, pp. 625-632, Jan. 2019.

63. L. Liu, W. Zhang, D. Wang, and Z. Peng, "Eventtriggered extended state observers design for dynamic positioning vessels subject to unknown sea loads," Ocean Engineering, vol. 209, no. 107242, Aug. 2020.

64. R. Cui, L. Chen, C. Yang, and M. Chen, "Extended state observer-based integral sliding mode control for an underwater robot with unknown disturbances and uncertain nonlinearities," IEEE Transactions on Industrial Electronics, vol. 64, no. 8, pp. 6785-6795, Aug. 2017.

65. R. Cui, L. Chen, C. Yang, and M. Chen, "Extended state observer-based integral sliding mode control for an underwater robot with unknown disturbances and uncertain nonlinearities," IEEE Transactions on Industrial Electronics, vol. 64, no. 8, pp. 6785-6795, Aug. 2019.

66. Z. Peng and J. Wang, "Output-feedback path-following control of autonomous underwater vehicles based on an extended state observer and projection neural networks," IEEE Transactions on Systems, Man, and Cybernetics: Systems, vol. 48, no. 4, pp. 535-544, Apr. 2018.

67. Z. Peng, D. Wang, T. Li, and M. Han, "Output-feedback cooperative formation maneuvering of autonomous surface vehicles with connectivity preservation and collision avoidance," IEEE Transactions on Cybernetics, vol. 50, no. 6 , pp. 2527-2535, Jun. 2019

68. Z. Peng, Y. Jiang, and J. Wang, "Event-triggered dynamic surface control of an under-actuated autonomous surface vehicle for target enclosing," IEEE Transactions on Industrial Electronics, vol. 68, no. 4, pp. 3402-3412, Apr. 2020.

69. M. Krstić, I. Kanellakopoulos, and P. Kokotovic, "Nonlinear and adaptive control design," John Wiley $\& 5$ Sons, 1995. 


\section{Supplementary Files}

This is a list of supplementary files associated with this preprint. Click to download.

- svjour3.cls

- svglov3.clo

- numcompress.sty

- model6numnames.bst

- model5names.bst

- model4names.bst

- model3numnames.bst

- model2names.bst

- model1anumnames.bst

- example.eps

- architectureheng.pdf

- ALLRefsY2020M09D04.bib

- 01203rdyrx1error.pdf

- 01202rdyx1error.pdf

- 3rdb3s32.pdf

- 3rdb2s22.pdf

- 3rdb1s12.pdf

- 2rdb2s2.pdf 